\title{
Effect of ventilation on the flowfield around a sphere
}

\author{
G. K. Suryanarayana, G. E. A. Meier
}

Abstract The flowfield around a sphere with and without ventilation was investigated in a wind tunnel over a range of Reynolds numbers in an incompressible flow. At supercritical $R e$, the pressure drag of a sphere can be nearly nullified by venting only $2 \%$ of the frontal area of the sphere to the base through a smooth internal duct. The drag reduction is achieved by increased pressures in the separated flow region close to the base. At high $R e$, the vent flow breaks through the near wake and brings about symmetry in the global flowfield. When the internal shear is increased by using a rough internal duct, the base pressure is unchanged, but the external flow is accelerated to velocities beyond that achieved by the potential flow around the basic sphere. The findings can be explained by a flow model in which the near wake is aerodynamically streamlined by a pair of counterrotating vortex rings at the base. A roughness element can be made to partially destroy the vortex system at the base and result in a steady asymmetric wake. A $1.2 \mathrm{~mm}$ diameter wire placed at $70^{\circ}$ was found to overtrip the boundary layer and completely destroy the vortex system.

Simultaneously, the turbulent separation is advanced and the drag increased.

At subcritical $R e$, ventilation marginally increases static pressures all over the surface. Since the large pressure

\section{Received: 7 July 1994/Accepted: 2 July 1995}

G. K. Suryanarayana

Experimental Aerodynamics Division, National Aerospace Laboratories, Bangalore, India

G. E. A. Meier

DLR Institute for Experimental Fluid Mechanics,

D-37073 Göttingen, Germany

Correspondence to: G. K. Suryanarayana

The work reported was carried out under a study grant from the German Academic Exchange Service (DAAD) in Bonn. The authors wish to thank the Director of DAAD in Bonn for the same. Thanks are due to Dr. F. R. Grosche and colleagues at DLR in Göttingen who assisted in the design, fabrication and wind tunnel testing of the sphere model. Thanks are also due to Prof. D. G. Mabey, visiting Professor, Imperial College, London for useful discussions. The many useful discussions with the research advisors of the first author viz., Dr. P. R. Viswanath of National Aerospace Laboratories and Prof. A. Prabhu of Indian Institute of Science, Bangalore are acknowledged with thanks. The support given by the Head, Experimental Aerodynamics Division, National Aerospace Laboratories is thankfully acknowledged. differential between the windward and leeward sides is not reduced, the internal flow has a rapid acceleration to a velocity close to that of the free stream. The reverse flow associated with the near wake forces the vent flow to rest within itself and the wake profile is unchanged. The main features of subcritical flow around the basic sphere are retained in spite of ventilation. The upstream effects of ventilation are greater for subcritical flow than for supercritical flow.

\section{1}

\section{Introduction}

Air flow around a bluff body such as a sphere or a cylinder causes a stagnation region on the windward side and a low pressure region surrounding the separated flow on the leeward side. When these two regions are interconnected or ventilated through an internal duct, a jet flow from the base is automatically set up by the external pressure distribution. The shear generated at the external and internal surfaces results in opposing vorticities. A constructive interaction between the two can result in a base pressure increase or a drag reduction.

The concept of ventilation is different from that of the well-known Base-bleed. Ventilation is a passive technique for a natural management of the pressure field around a bluff body and involves a feedback of downstream flow changes to the upstream flow parameters. Further, the magnitude of the jet velocity at the base is of the order of free stream velocity. In base bleed, the bleed velocity is usually a fraction of the free stream velocity in order that the device drag is small. Base bleed results in a forced flowfield governed by the energy input into the system. Wood $(1964,1967)$ and Bearman (1967) have studied the effect of base bleed on an airfoil with a blunt trailing edge. According to them, base bleed delays the onset of instabilities in the shear layer and thereby increases the vortex formation distance. The delay is shown by dimensional analysis to result in a base pressure increase and a consequent drag reduction. This effect was found to be greater when the width of the jet is large as compared to that of the base. The base pressure monotonically increased up to a certain value of the blowing moment coefficient and for higher blowing the effect was negative. More recently, Zhdanov and Eckelmann $(1990,1991,1992)$ have observed that the maximum base pressure achieved is a function of the bleed rate as well as width of the jet. For models with narrow slot, the maximum base pressure occurs at much the same momentum flux density of the jet as for wider slots and reports that thick and thin jets may not have identical effects. Wong (1985) has reported 
stabilisation of the wake by base bleeding a cylinder in a water tunnel. In this experiment, the stagnation flow around a hollow cylinder was vented into its base using a concentric inner cylinder at $R e$ varying from 2000 to 3000 . Measurement of forces was not made. In this experiment however, the internal flow passes around an annulus and undergoes large pressure changes resulting in a large reduction in the vent mass flow which is an important parameter.

The concept of ventilation of a sphere was first reported by Meier et al. (1990) in which a drag reduction of the order of $10 \%$ was observed from tests on vented spheres in free fall at a subcritical $R e$. It was also observed from interferograms of flow around vented cylinders that a divergent internal vent collapses the near wake into a twin-eddy like structure at moderate Re. A convergent or parallel internal vent resulted in a flip-flop behaviour of the jet inside the near wake. Drag reduction of the order of $50 \%$ to $60 \%$ due to ventilation at supercritical $R e$ from direct force measurement in a wind tunnel was reported by Meier et al. (1991) and Suryanarayana et al. (1992). At low (subcritical) Re, no significant drag reduction was observed. Results from force measurements and flow visualisations are reported by Suryanarayana et al. (1993) based on wind tunnel and water tunnel experiments. A drag reduction of $50 \%$ to $60 \%$ was measured for parallel, convergent or divergent internal vents. A possible model of the flow at high $R e$ around a vented sphere was also proposed. It was reported that venting at high $R e$ has the effect of aerodynamically streamlining the base, like a splitter plate. It must however be noted that efforts to find a three-dimensional equivalent of a splitter plate behind a two-dimensional cylinder have not succeeded. Maxworthy (1969) introduced a cylinder in the wake of a sphere and found that the base pressure did not change until the cylinder was moved very close to the base. This is because of the fact that the three-dimensional flow allows for circumferential communication of pressure signals unlike a two-dimensional flow.

Dimensional analysis of the flow around the vented sphere shows that the drag depends on $\operatorname{Re},(\Delta P / q)$ and $(d / D)$ where $\Delta P$ is the differential pressure across the duct, $q$ the dynamic pressure, $d$ the diameter of the internal duct, $D$ the diameter of the sphere and $R e$ the Reynolds number based on $D$. In the earlier experiments of Suryanarayana et al. (1993), values of 0.15 and 0.10 for the ratio of diameters were found to result in the same amount of drag reduction. Since the present work was aimed at verifying the earlier results by pressure measurements rather than optimise the diameter ratio, a value of 0.15 was chosen for the tests.

The present work is mainly an extension of the earlier work of Suryanarayana et al. (1993) and hence an extensive reference of the same will be made. The main objective was to obtain the pressure distribution on the sphere with and without ventilation over a range of $R e$. Additionally, the development of flowfield around the vented sphere, wake profiles and internal velocity profiles were also obtained. In this paper, the results will be discussed and compared with the data available in the literature on the basic sphere. Pressure distribution on the basic sphere was obtained by closing the vent holes using a curved flat plate at the entry and an adhesive tape at the rear. The work reported forms a part of the doctoral thesis of the first author.

\section{Description of the vented sphere, experimental set-up and instrumentation}

The vented sphere consists of two asymmetric parts turned out of Aluminium alloy. The bigger part is provided with a smooth entry which is matched to an internal duct open at the base. The smaller part can be fixed to the bigger one as a covering shell and rigidly fixed. Provision is made in the bigger part to accommodate a scanivalve and a supporting sting. When the two parts are assembled, the vented sphere has an outer diameter of $200 \mathrm{~mm}$. The internal diameter of the duct is $30 \mathrm{~mm}$. A final polishing after assembly of the two parts provided a very smooth surface with a mirror finish. The vented area corresponds to $2.25 \%$ of the frontal area of the sphere. Figure 1 shows the constructional details and a schematic of the model which was designed using CATIA Software at the DLR Institute of Experimental Fluid Mechanics, Göttingen, Germany.

The sphere was asymmetrically fixed to a support sting at a location of $150^{\circ}$ from the stagnation point of the basic sphere as shown in Fig. 1. The sting has a diameter of $10 \mathrm{~mm}$ up to a distance of $100 \mathrm{~mm}$ behind the sphere and then increases to $25 \mathrm{~mm}$ up to a length of $600 \mathrm{~mm}$. Justification for asymmetric mounting of the sphere is included along with the results and discussions. The sting was held at the downstream end using a heavy sector arrangement fixed rigidly to the ground.

Distance between the base of the sphere and the sector was nearly $600 \mathrm{~mm}$.

Experiments were conducted in the $1 \mathrm{~m}$ wind tunnel at DLR. The wind tunnel is a closed circuit open jet facility driven by a propeller with variable speed control. A nozzle with a rectangular exit of dimensions $0.75 \mathrm{~m} \times 1 \mathrm{~m}$ provides the jet flow. Length of the test section is about $1.25 \mathrm{~m}$ and the free stream turbulence intensity $(\mathrm{Tu}) 0.15 \%$. The maximum free stream velocity is $55 \mathrm{~m} / \mathrm{s}$. A traverse mechanism can be fixed on a truss work above the test section to move a probe to any desired position within the test volume. Figure 2 shows a photograph of the vented sphere installed in the test section of the $1 \mathrm{~m}$ wind tunnel. Thirty holes of diameter $0.4 \mathrm{~mm}$ were drilled at various locations normal to the surface along a spiral for measurements of surface pressures as shown in Fig. 1. The spiral shape was chosen to avoid any interference among pressure ports. Helical symmetry in the flow was assumed for interpretation of results. Ten holes of the same size were drilled on the internal duct for measurement of wall pressures. All the pressure ports were connected to a 48 port Scanivalve manufactured by Pressure Systems Inc., USA. Static pressures were measured using a SETRA pressure transducer of range $140 \mathrm{~mm}$ of water ( $13.5 \mathrm{mBar}$ ) with atmospheric pressure as the reference. The free stream velocity was determined from the pressure difference between the atmosphere and the settling chamber using a similar transducer of range $127 \mathrm{~mm}$ of water (12.3 mBar). A $1.2 \mathrm{~mm}$ wire rolled into a ring was fixed at $70^{\circ}$ in order to trip the boundary layer.

A static pressure tube of diameter $3 \mathrm{~mm}$ and length $370 \mathrm{~mm}$ was introduced from the rear vent hole using the traverse mechanism and traversed along the centreline from upstream to downstream for measurement of static pressures. A SETRA transducer of range $63.5 \mathrm{~mm}$ of water $(6.1 \mathrm{mBar})$ with atmospheric reference pressure was used for this purpose. For measurements of internal velocity profiles, a $3 \mathrm{~mm}$ diameter 

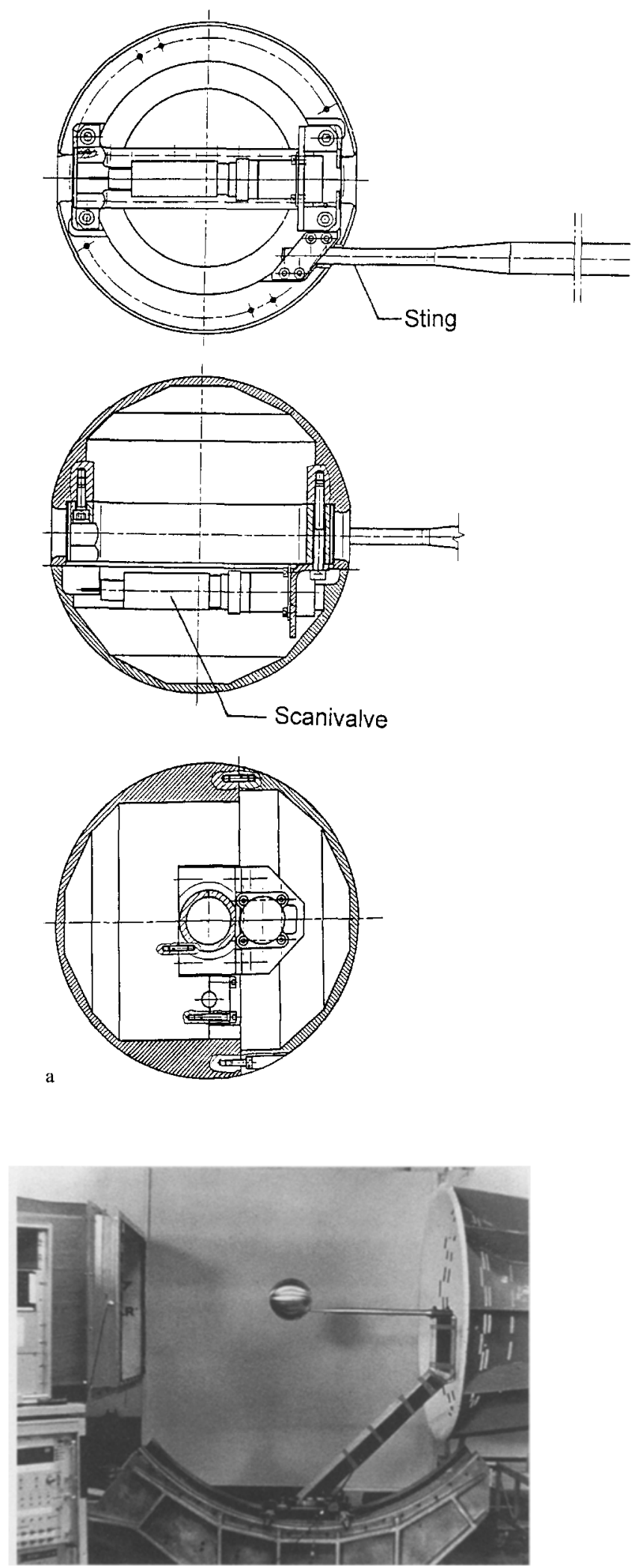

Fig. 2. Installation photograph of the vented sphere model

Prandtl tube was positioned $50 \mathrm{~mm}$ upstream of the base inside the sphere using the traverse mechanism. Total pressure obtained from the tube and the wall static pressure measured on the duct were used for measurement of velocity. The probe
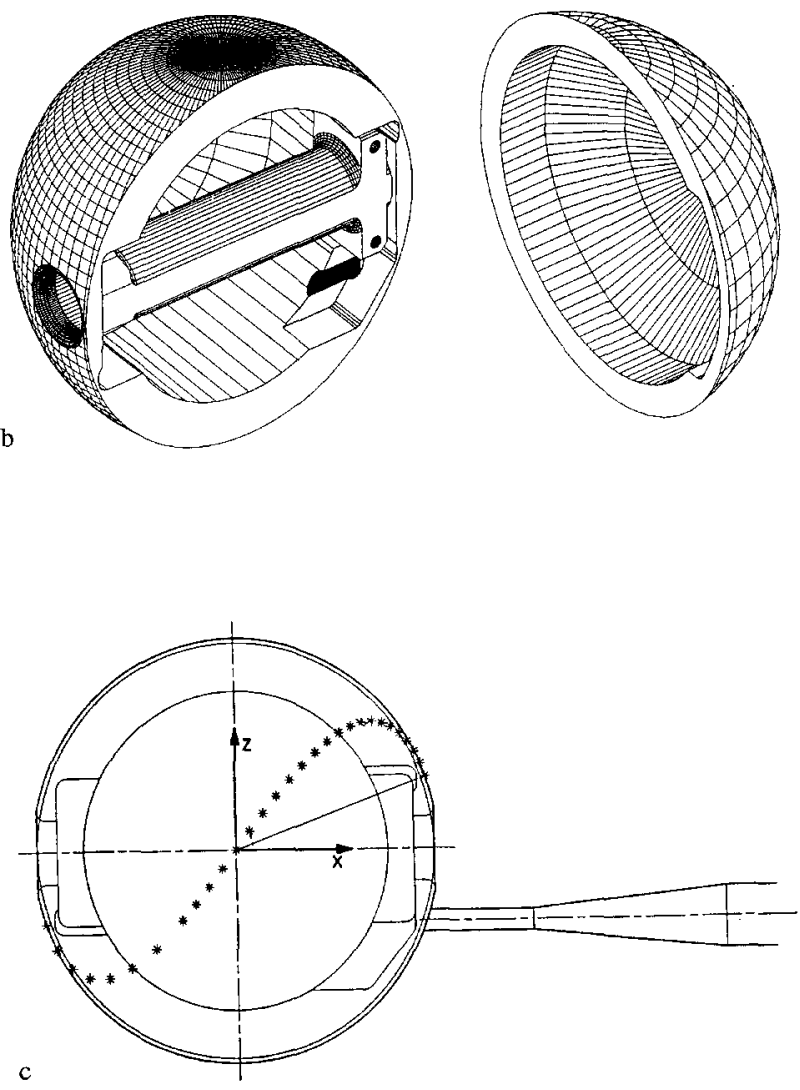

Fig. 1a-c. Constructional details and distribution of pressure ports. a Model constructional details; $\mathbf{b}$ isometric view of the vented sphere; $c$ distribution of pressure ports

was also traversed along horizontal and vertical planes inside the duct. Total pressure was measured using a SETRA transducer of range $140 \mathrm{~mm}$ water $(13.5 \mathrm{mBar})$ and static pressures measured using a similar transducer of range $63.5 \mathrm{~mm}$ water $(6.1 \mathrm{mBar})$. The Prandtl tube was traversed along the downstream centreline up to two sphere diameters and pressures recorded. Wake of the sphere was scanned using the Prandtl tube at two diameters behind the base along a horizontal plane. A delay time of 4 seconds was chosen for the scanivalve ports and the pressure signals from the static and total pressure tubes were integrated over 10 seconds.

\section{3}

\section{Results and discussions}

\section{1}

Effect of ventilation on the pressure distribution

Figure 3 shows the pressure distribution obtained with (vented sphere) and without venting (basic sphere) the sphere at subcritical $R e$ and comparison of the data on the basic sphere from literature. It is apparent that venting leads to increased 


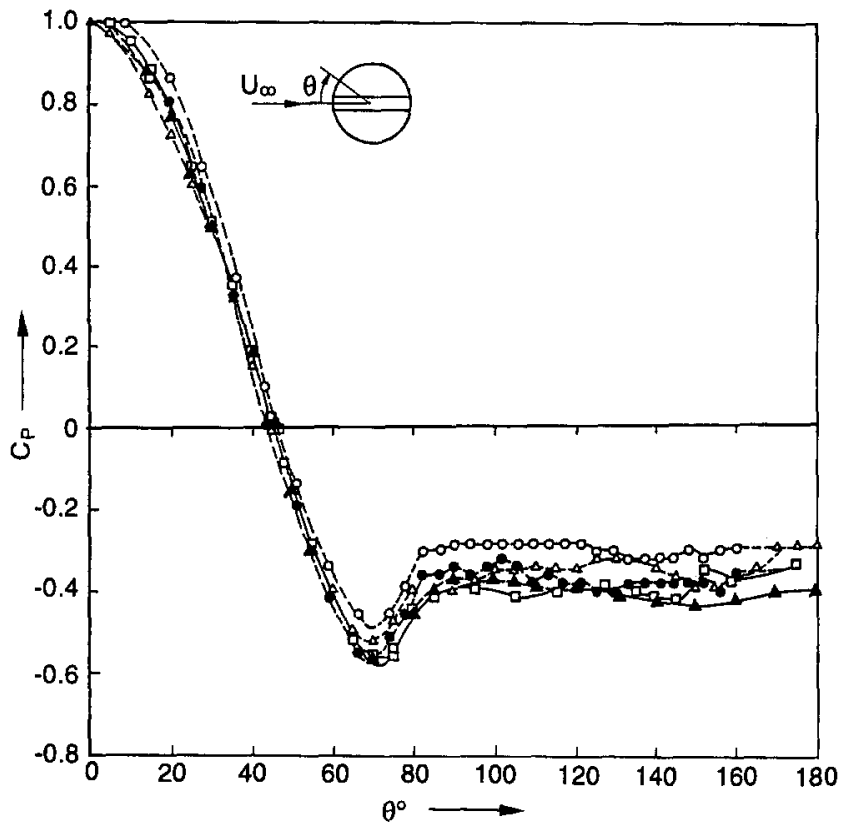

\begin{tabular}{|c|c|c|}
\hline Symbol & Configuration & Description \\
\hline 0 & Vented Sphere & Present Result at Re $=0.6 \times 10^{5}$ \\
\hline$\bullet$ & Basic Sphere & Present Result at Re $=0.6 \times 10^{5}$ \\
\hline$a$ & Fage (1936) & Re $=1.1 \times 10^{5}$ \\
\hline$\Delta$ & Maxworthy $(1969)$ & Re $=0.6 \times 10^{5}$ \\
\hline$\Delta$ & Achienbach $(1972)$ & Re $=1.62 \times 10^{5}$ \\
\hline
\end{tabular}

Fig. 3. Effect of venting on pressure distribution at subcritical $R e$

pressures all over the surface, from the stagnation ring at the front $\left(8.2^{\circ}\right)$ to the base $\left(171.2^{\circ}\right)$. Thus, a decrease in drag as a result of base pressure increase is nearly nullified by increased drag due to windward static pressures. Thus, a ventilation of the present kind is unlikely to reduce the drag at low $R e$.

Figure 4 shows the pressure distribution at supercritical $R e$ with and without venting the sphere and comparison with data from literature on the basic sphere. Venting leads to increased pressures on the windward side almost up to $90^{\circ}$. A laminar separation bubble occurs over nearly $10^{\circ}$ after about $100^{\circ}$, as evidenced by the plateau in the pressure curve of both the vented and basic spheres. Reattachment of turbulent shear layer occurs at about $110^{\circ}$ and a rapid pressure recovery takes place thereafter. The occurrence of laminar separation bubble in this range of $R e$ can be noticed in the oil-flow pattern also. The vented sphere shows lower pressures (or increased velocities) up to about $125^{\circ}$ as compared to the basic sphere. The most interesting feature occurs beyond $125^{\circ}$ when venting increases the pressure and makes the pressure distribution in the base region uniform and positive. Beyond the turbulent separation on the basic sphere at $135^{\circ}$, the separated flow shows large pressure gradients. Venting is seen to marginally delay the turbulent separation.

According to Taneda (1978), the separated turbulent shear layer behind a sphere at high $R e$ rolls up into a threedimensional horse shoe vortex structure with a pair of counter rotating streamwise vortices as shown in Fig. 5. The entire structure rotates randomly about a streamwise axis. As a result, the basic sphere at high $R e$ experiences an unsteady aerodynamic force whose direction is arbitrary. Dallmann and Schewe (1987) have identified the various critical points

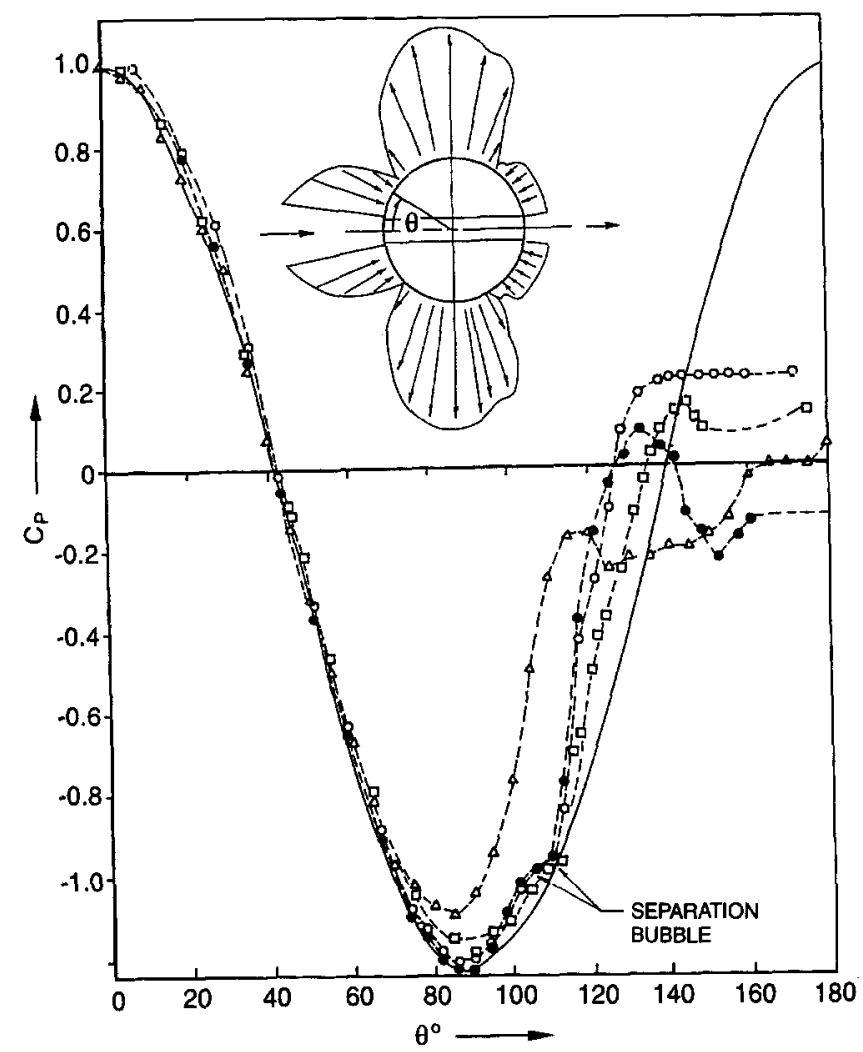

81

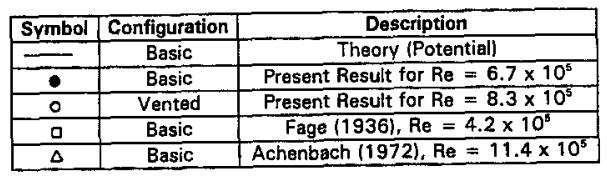

Fig. 4. Effect of venting on pressure distribution at supercritical $R e$

associated with such a flow based on topological considerations of three-dimensional separated flows. According to Suryanarayana et al. (1993), ventilation of the sphere at high $R e$ causes the separated shear layer to roll up as a base vortex ring primarily due to its entrainment by the secondary vortex ring which is formed by the vent flow close to the base as shown in Fig. 5. The combined effect of the vortex rings is to provide an aerodynamic streamlining at the base. The occurrence of a pressure plateau in the separated flow region as in a subcritical flow confirms the absence of large scale flow oscillations in the wake as visualised on a laser light sheet in the referred work. Figure 6 shows the increase of base pressure (at the last measurement point at $160^{\circ}$ ) due to venting as compared to that of the basic sphere from the present measurements over a range of $R e$.

Integration of the pressure distribution

$\left(C_{d}=\int_{8.2}^{17.8} C p \sin 2 \Theta d \Theta\right)$ shows that in the supercritical range of $R e$, the pressure drag of the vented sphere is nearly zero. This is because of the base pressure increase (base thrust) which nearly compensates the drag due to windward static pressures as shown by the pressure distribution in Fig. 4. The net drag on the vented sphere would then be due to skin friction due to internal and external flows and a pressure drag due to the radius of the vent hole, all of which are expected to be very small. 

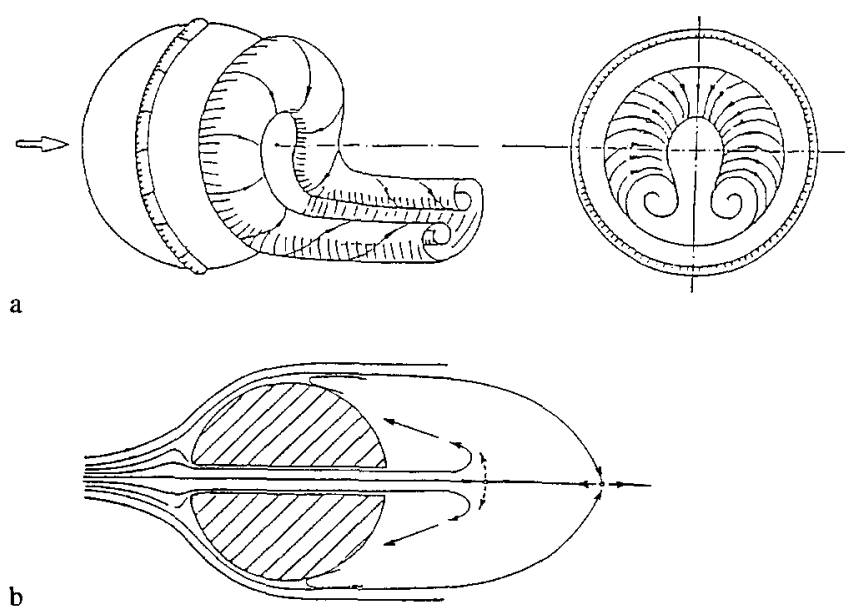

b

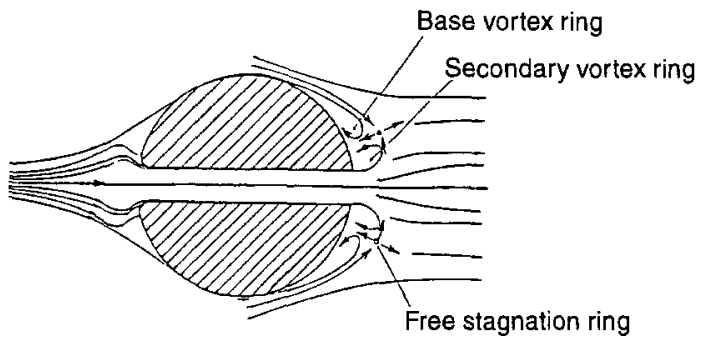

Fig. 5a-c. Structure of the wake behind a sphere with and without venting. a Basic sphere at high $\operatorname{Re}$ (Taneda, S., J.F.M. 85, 1978); b vented sphere at subcritical $R e$; $\mathrm{c}$ vented sphere at supercritical $R e$

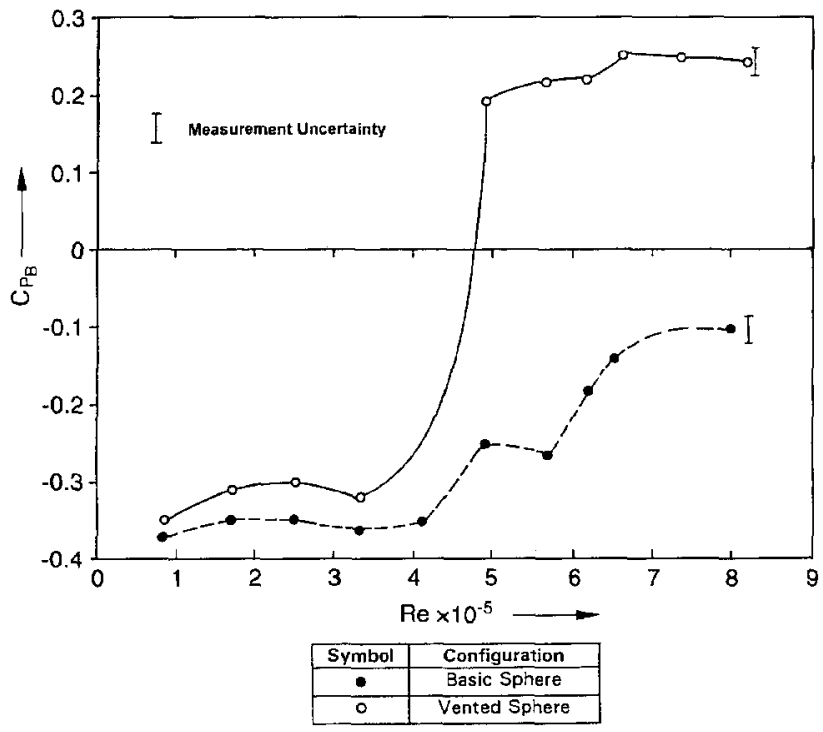

Fig. 6. Variation of base pressure with $R e$ for basic and vented spheres

The earlier study of Suryanarayana et al. (1993) reported a drag reduction of $50 \%$ to $60 \%$ in the supercritical range and hardly any change at lower $R e$ from direct measurement of force in a wind tunnel using an external balance. Correction due to the support system was estimated by supporting the sphere from an alternate support through which the effect of the sphere wake on the original support system was identically simulated. It was assumed that the correction factor would be the same for both the vented and the basic spheres. The present measurements indicate that the pressure drag, which corresponds to nearly 90 to $95 \%$ of the total drag (Achenbach, 1972 ) is nullified. The discrepancy is due to underestimation of the support drag in the earlier work when the sphere was vented. Aerodynamic streamlining due to ventilation results in higher dynamic pressures in the wake and hence the tare drag correction due to the support system would be higher for the vented sphere than that for the basic sphere. If the same correction factor is used, the resulting figure is an underestimate of the actual reduction achieved.

3.2

\section{Comparison of results on the basic sphere}

Figures 3 and 4 also show comparisons of the present results on the basic sphere with those of Fage (1936), Maxworthy (1969) and Achenbach (1972) at subcritical and supercritical $R e$ respectively. The discrepancies in the results can be attributed to four factors viz., small variations in $R e$, model support position, $T u$ and the model surface roughness. Each of these factors can play an important role as discussed below:

When the separated laminar shear layer starts reattaching on the surface, the drag curve starts sinking and reaches the minimum drag condition. At higher $R e$, the drag starts increasing again. Therefore, the pressure distribution can show large changes even for small changes in velocity in this range of Re.

A reasonable agreement is seen between the present data and that from literature as shown in Fig. 3, even though the present results are for an asymmetric support whereas the others except Maxworthy (1969) are all for symmetric rear supports. This observation is consistent with that of Raithby and Eckert (1968) who have studied the effects of a cross flow support and a conventional rear support on the surface flow over a sphere. This feature is essentially because of the fact that in subcritical flow, separation of the laminar boundary layer occurs at about $82^{\circ}$ without a turbulent reattachment. A support fixed downstream of it lies in the separated flow region and does not strongly influence the windward flow. Since the present support system is at $150^{\circ}$ which is well downstream of laminar as well as turbulent separation, it does not have a significant influence on the results. Further, in the earlier experiments, it was found that the reduction in drag due to ventilation was unaffected when the vented sphere was rotated about the asymmetric sting axis.

Figure 4 shows comparison at supercritical Re with those of Fage (1936) and Achenbach (1972). Good agreement is shown with the data of Fage almost up to the point of turbulent reattachment of the flow. Turbulent separation is indicated at about $145^{\circ}$ in Fage's results whereas at about $135^{\circ}$ in the present results. Whereas a clear pressure gradient in the separated flow region is indicated in the present results, data in the region from $150^{\circ}$ to $175^{\circ}$ are not reported by Fage. The data of Achenbach (1972) which are for a slightly higher $R e$ agree well with the present data only up to $70^{\circ}$. The value of minimum $C P$ achieved is higher than that shown by the present data as well as that by Fage (1936). Large differences are shown by Achenbach's results at locations beyond $90^{\circ}$, apparently because of the higher $R e$ as well as $T u$ of $0.45 \%$ as compared to the $T u$ of $0.15 \%$ for the present results. This is also suggested by the absence of laminar separation bubble and the advanced 
separation at $120^{\circ}$. The locations of laminar separation, turbulent reattachment and turbulent separation in the present tests are in good agreement with the visual observations of Taneda (1978) in a wind tunnel of $T u 0.12 \%$.

The effect of surface roughness on the flow past spheres has been studied by Achenbach (1974). Roughness elements on the surface can cause earlier transition, advance the turbulent separation and create wider wakes depending on the roughness size. Consequently, spheres with different surface roughness in wind streams of varying values of $T u$ can show variations in the locations of flow separation and reattachment. In the present tests, the effects of ventilation have been obtained from tests on the same sphere under identical free stream and model support conditions by opening and closing the vent holes. It is therefore believed that the uncertainties as described above do not affect the relative changes observed due to ventilation.

\section{3}

\section{Effect of tripping the internal flow}

The vent flow emerging from the base shows a laminar character at $R e$ less than about 2000 as reported by Suryanarayana (1993). At higher $R e$, the edge of the jet becomes turbulent even when the internal duct is smooth. Experiments were done by tripping the internal flow using a P400 sandpaper which was fixed over a length of $30 \mathrm{~mm}$ upstream from the base. Fig. 7

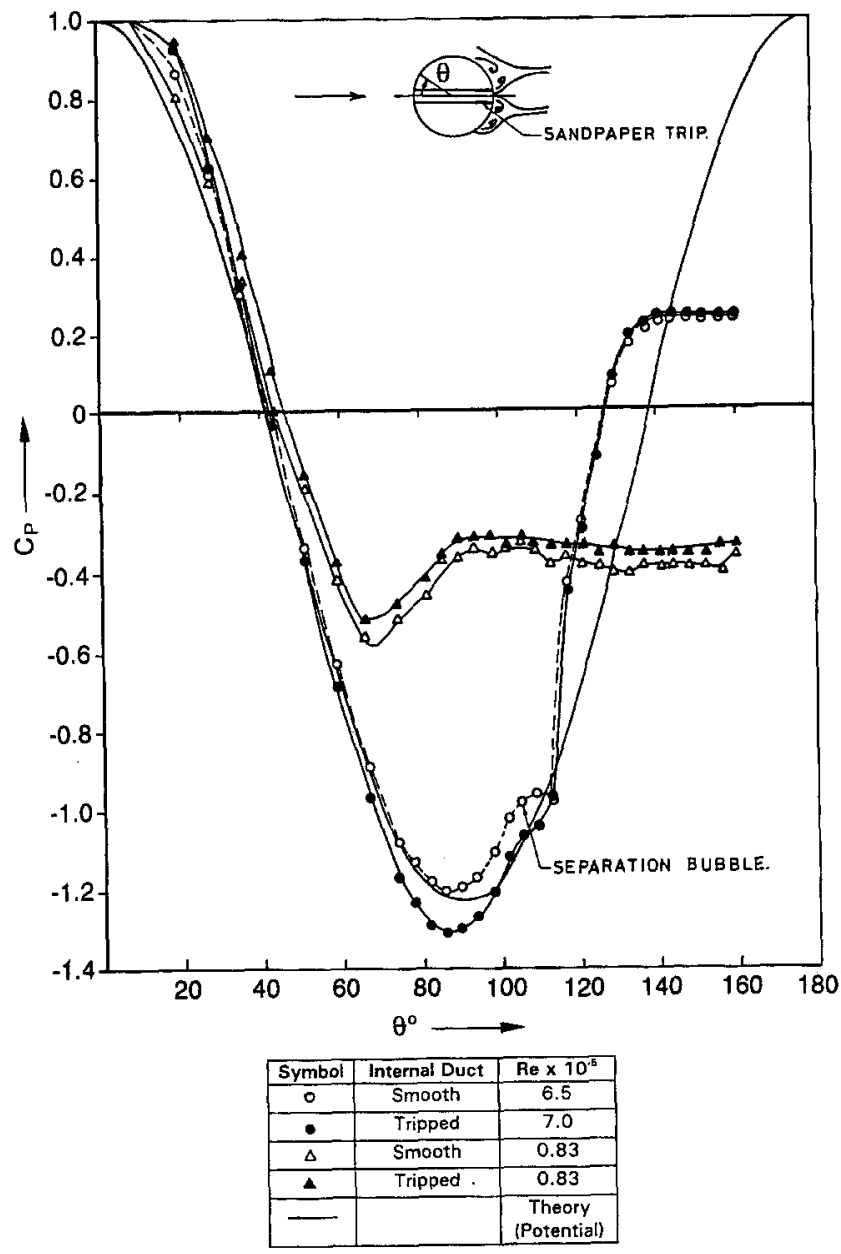

Fig. 7. Effect of tripping the internal flow on the external pressure distribution around the vented sphere shows the pressure distributions obtained with smooth and tripped internal ducts. At subcritical $R e$, the tripped duct causes increased pressures all over the surface. The magnitude of increase is greater on the windward side than on the leeward side up to about $40^{\circ}$. Accordingly, no significant change in drag may be expected. At supercritical $R e$, the windward pressures up to $40^{\circ}$ are higher than for the smooth duct, but lower in the region $50^{\circ}$ to $120^{\circ}$. Interestingly, the pressures in the region $50^{\circ}$ to $100^{\circ}$ are even lower than the potential values for the flow over the basic sphere. In addition, the laminar separation bubble which occurred on the external surface with the smooth duct seems to have either diminished or vanished when the duct is tripped. The base pressures are however not affected.

When the internal duct is tripped, the shear generated at the edge of the jet and hence the entraining action of the secondary vortex ring (Fig. 5) is enhanced. As a result, the base vortex ring increases the local accelerations on the external surface. Consequently, the boundary layer gets thinner and resists laminar separation. Since the base pressures are not much altered, the near-zero pressure drag condition is unaffected when the internal duct is tripped.

\section{4}

\section{Effect of disturbances and boundary layer tripping wire on the external flow}

A roughness element (an adhesive tape $15 \times 4 \times 0.2 \mathrm{~mm}$ ) on the external surface at supercritical $R e$ can generate disturbances in the surface flow which break through the separation bubble causing an advanced local separation and partially destroy the base vortex system. Vorticity conserved within the recirculation zone is then convected into the wake by a pair of counter rotating streamwise vortices from specific locations behind the vented sphere. Figure 8 shows the effect of the roughness element on the pressure distribution at supercritical $R e$ and a corresponding oil-flow pattern. The loss of base pressure increase and increase of drag are apparent. It is interesting to note that as a result of the roughness element, pressure gradients reappear in the separated flow region, as was the case with the basic sphere. The structure of the wake may then be expected to be similar to that of the basic sphere. An identical observation was made by Suryanarayana et al. (1993) by visualising the wake on a laser light sheet. The main difference would be that this wake does not randomly oscillate. As a result, the model may be expected to experience a steady asymmetric side force.

Figure 9 shows the effect of a $1.2 \mathrm{~mm}$ diameter boundary layer tripping wire fixed at $70^{\circ}$ from the centre on the pressure distribution at subcritical $R e$. Results for free transition are also shown. The trip wire appears to cause a local separation ahead of itself and introduce a large velocity increment. Boundary layer separation is delayed from $82^{\circ}$ to about $120^{\circ}$. A small increase of base pressure as compared to the basic sphere is also observed.

Figure 10 shows the effect at supercritical $R e$. The effect is identical to that at subcritical Re. Additionally, the turbulent separation is advanced from $135^{\circ}$ to $125^{\circ}$, base pressure recovery lost and the drag reduction disappears. The adverse effects are apparently caused by overtripping of the boundary layer by the wire. $R e$ based on the trip height varies from 2000 to 5000 in the present tests. The earlier results of 

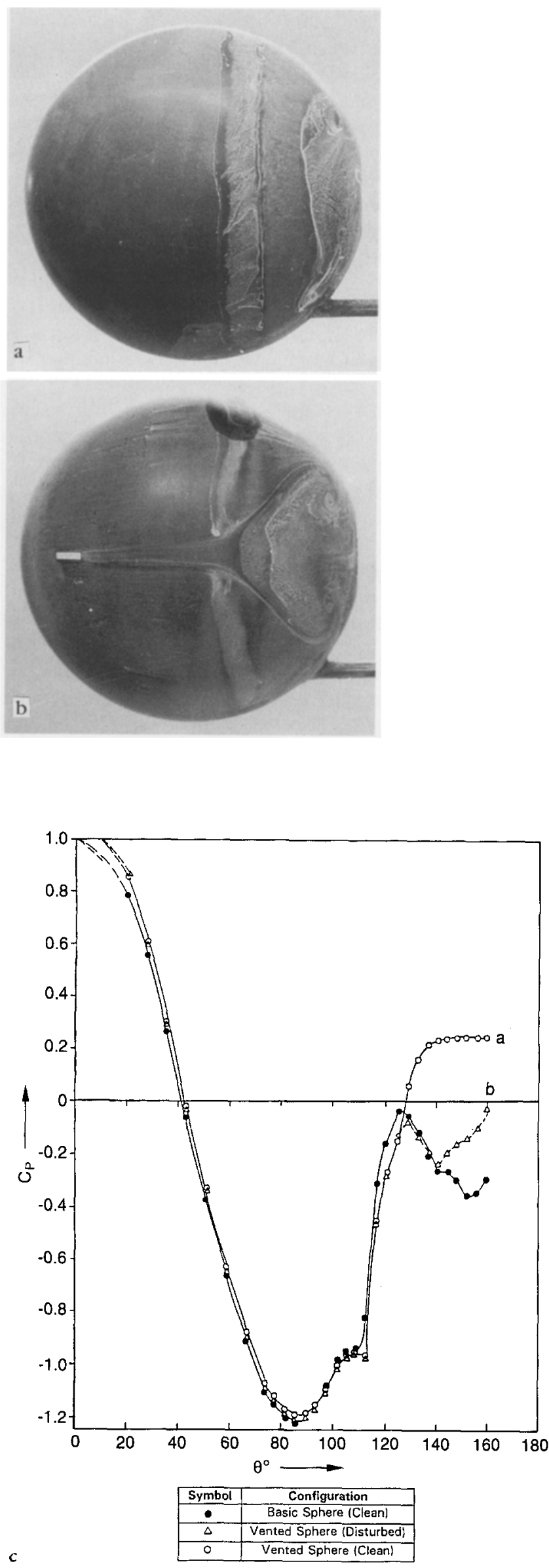

Suryanarayana et al. (1993) showed that the tripped vented sphere had no significant drag reduction as compared to the tripped basic sphere. As a result of earlier separation (wider wake) and also because of the additional turbulent kinetic energy introduced in the separated shear layer, the mechanism causing the base pressure rise is adversely affected. The structure of the wake is expected to be the same as at a subcritical $R e$, in that the internal flow dissipates within the near wake.

Separation of turbulent boundary layer on the vented sphere occurs at about $140^{\circ}$. The separated shear layer is then at its closest location with respect to the edge of the internal jet flow. As a result, a strong mutual interaction is facilitated. When the internal vent geometry is varied, this interaction distance is also varied. When the vent has a divergence, the distance is lesser than when it is parallel or convergent. However, the force measurements (Suryanarayana et al. 1993) indicated identical drag reduction for all the vent geometries. It is possible that the vent area ratio of $2.25 \%$ chosen was too high to observe this

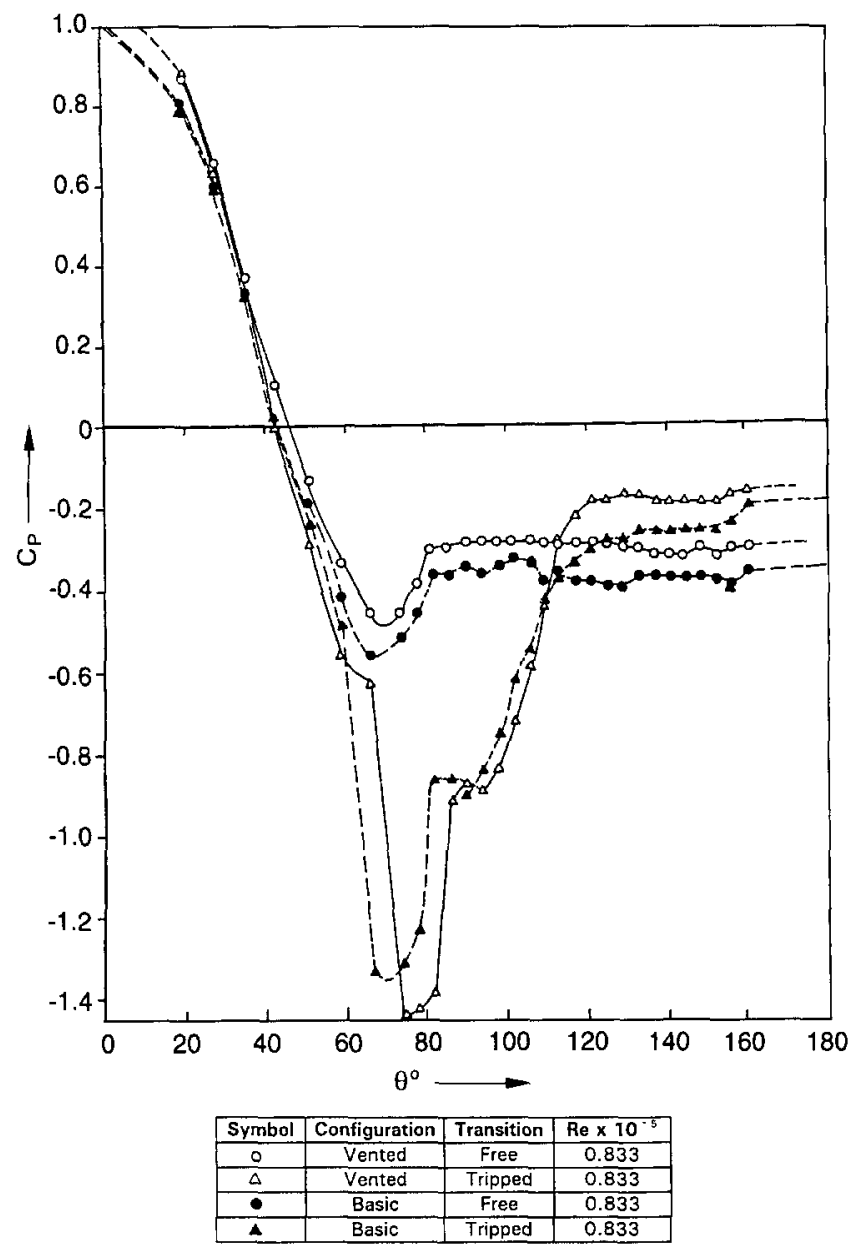

Fig. 9. Effect of boundary layer trip wire on pressure distribution at subcritical $R e$

Fig. 8a-c. Effect of roughness element on the flow around the vented sphere at supercritical $\operatorname{Re}\left(5.8 \times 10^{5}\right)$. a Clean model, b with a roughness element, $c$ Distortion of pressure distribution due to partial destruction of the base vortex system 
effect. In contrast, interferograms of flow around vented (slotted) cylinders show a large reduction in the width of the wake and occurrence of attached twin-eddy like structure when the vent is divergent as compared to unchanged wake width with parallel or convergent vent (Meier et al. 1990).

\section{5}

\section{Internal flow measurements}

Figure 11 shows the pressure distributions along the internal duct of the vented sphere at subcritical and supercritical $R e$. Also shown are the pressures when the external and internal flows are tripped. Because of the boundary layer growth inside the duct, the internal flow is accelerated.

The acceleration is greater at subcritical than at supercritical $R e$ because of greater $\Delta P$ at lower $R e$. The higher acceleration results in negative wall pressures and the reduced acceleration results in positive wall pressures. In both ranges of $R e$, the magnitude of wall pressure reduces with $R e$ whereas the wall pressure slope is nearly constant in each range. The wall pressure distributions in subcritical and supercritical ranges follow different laws. Figure 11 also indicates linear curve fits obtained under various internal and external flow conditions.

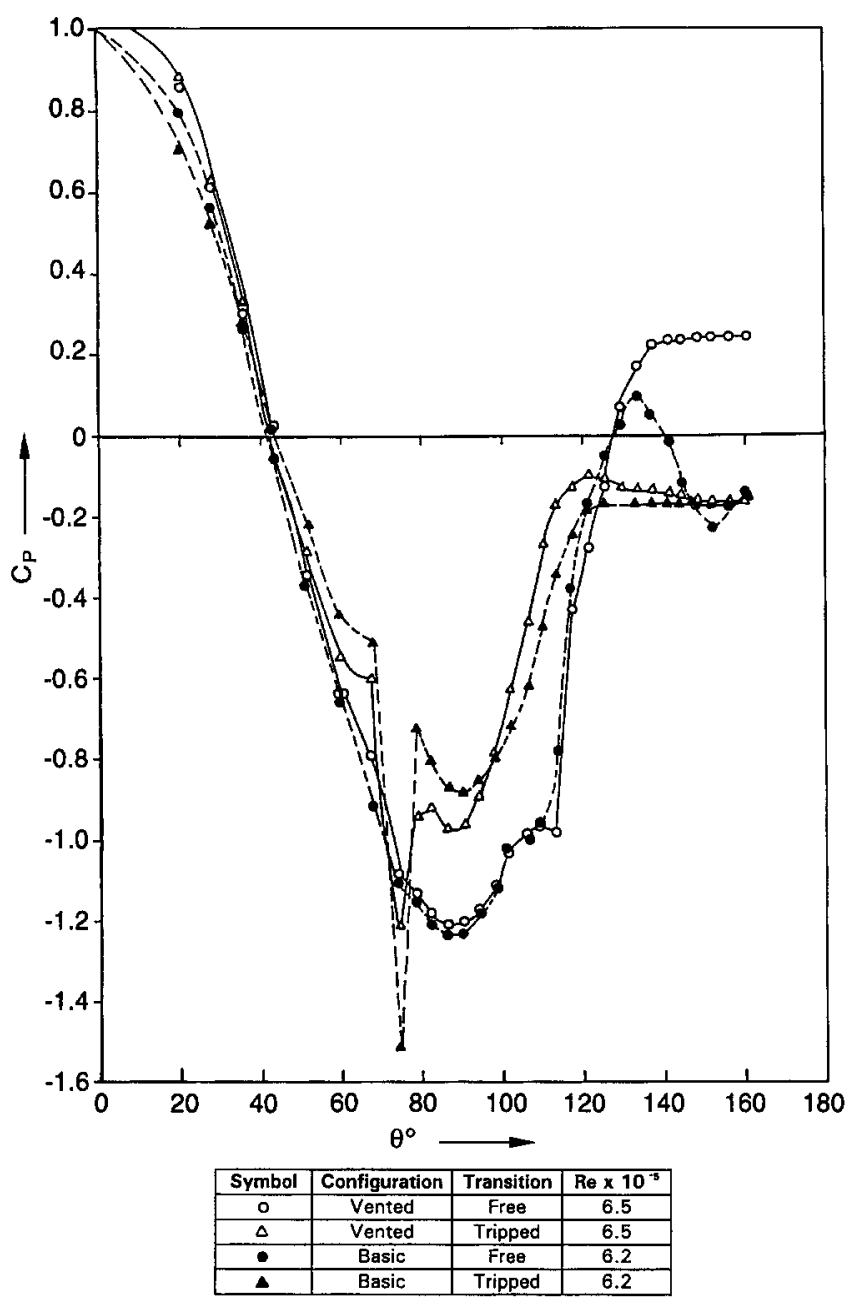

Fig. 10. Effect of boundary layer trip wire on pressure distribution at supercritical $R e$
When the external flow is tripped at a high $R e$, the decreased base pressure results again in higher acceleration. When the internal duct is tripped at a high $R e$, the wall pressures show an increase over the smooth duct case even though the base pressures were not further increased. This suggests that the mechanism responsible for the base pressure rise is more sensitive to disturbances in the external flow than in the internal flow. Therefore, a symmetric support located in the middle of the vented sphere is unlikely to invalidate the present observations.

Figure 12 shows typical velocity profiles measured at subcritical and supercritical $R e$ across the duct. The measurement location was at $0.82 D(5.4 d)$ from the entrance to the vent at a station where the wall static pressure data could be obtained separately (at $(x / D)=0.32)$ as described in 2.0 above. Static pressures across the duct along horizontal and vertical diameters at this station as well as elsewhere in the duct were constant and equal to the wall pressure, except when very close to the entrance. The profiles are typical of a developing pipe flow, which must be expected considering the length of the duct. Mean velocity in the duct at the measuring station is obtained by integrating the profile assuming no slip at the walls.

Mean velocity in the duct reduces to $0.693 U_{\infty}$ at higher $R e$ as against $0.919 U_{\infty}$ at lower $R e$ due to increased base pressures.

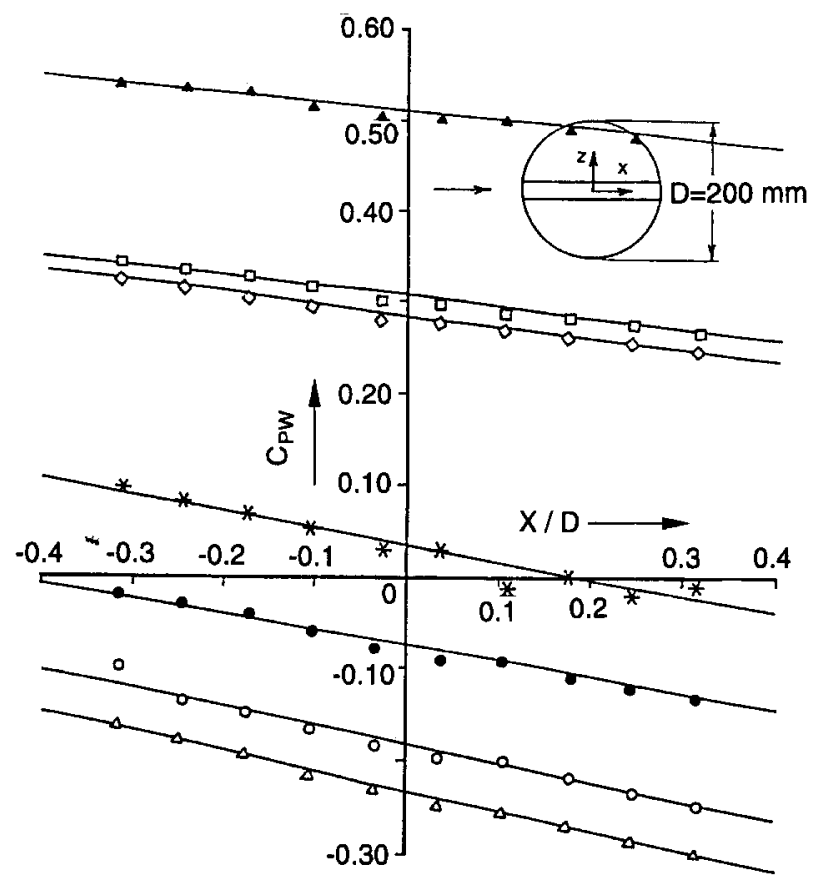

\begin{tabular}{|c|c|c|c|c|}
\hline Symbol & Ext. Flow & Int. Flow & Re x 10 & Cpw Vs $\{x / D)$ \\
\hline 0 & Smooth & Smooth & 0.833 & $-.1836-.2168(x / D)$ \\
\hline$\Delta$ & Smooth & Smooth & 3.250 & $-.2377-.2181(x / D)$ \\
\hline$D$ & Smooth & Smooth & 6.550 & $.3020-.1240(x / D)$ \\
\hline 0 & Smooth & Smooth & 8.160 & $.2835-.1260(x / D)$ \\
\hline$\bullet$ & Tripped & Smooth & 6.530 & $-.0766-.1808(x / D)$ \\
\hline$*$ & Smooth & Tripped & 1.560 & $.0310-.1930(x / D)$ \\
\hline$\Delta$ & Smooth & Tripped & 7.030 & $.5090-.1040(x / D)$ \\
\hline
\end{tabular}

Fig. 11. Pressure distribution on the internal duct of the vented sphere 


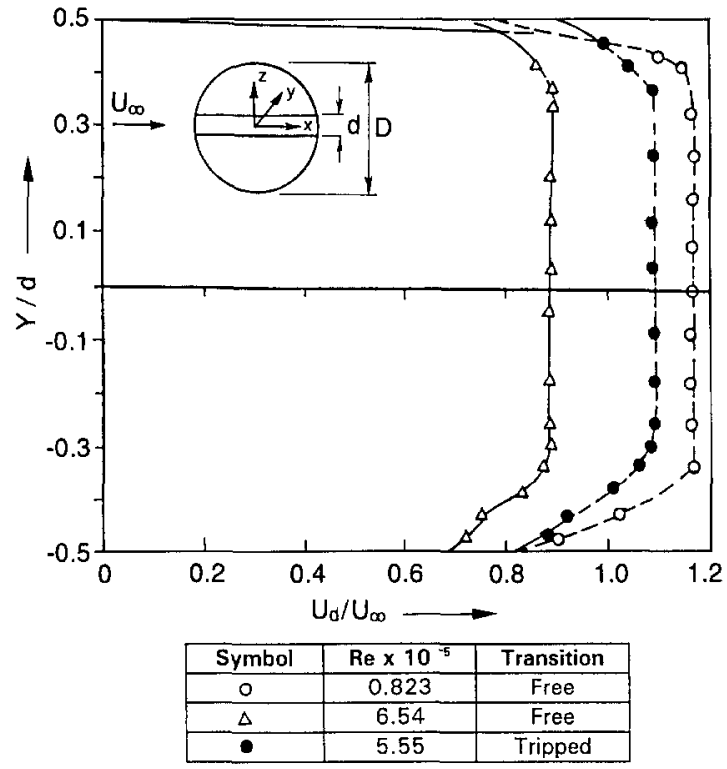

Fig. 12. Internal velocity profiles at $(x / D)=0.32$

When the internal flow is tripped at high $R e$, the mass flow inside the duct reduces further as compared to the smooth duct case, but the base pressure recovery and the drag reduction are unaltered. Hence, if the diameter of the internal duct is reduced from the present value, the mean quantities are unlikely to change unless the diameter is too small when viscous effects predominate.

\section{6}

\section{Static pressure variation along the centreline}

Figure 13 shows the variation of static pressure along the centreline of the vented sphere from about half a diameter upstream of the front to two diameters downstream of its base. The upstream pressures were measured using a static pressure tube whereas the downstream pressures were measured using a Prandtl tube. A few data points in the overlapping region are also shown. In both the Re ranges, the upstream pressure reaches a maximum of $C p \cong 0.75$ at a distance of $0.13 D$ ahead of the front and then drops rapidly as the entrance is approached. The occurrence of maximum is because of the greater upstream influence of the sphere as compared to that of the vent hole. Fluid particles approach the sphere as if there was no vent hole and reach a maximum $C p$ before accelerating into the duct. Further, since the stagnation ring occurs normal to the external surface, the streamlines must diffuse close to the entrance as shown in Fig. 5. Minimum pressure in the base occurs at $0.35 D$ downstream of the base. As noted by Roshko (1954), minimum pressure in the base region is in the core of the separating vortex downstream of the base. After reaching the point of minimum pressure, the flow gradually reaches the ambient conditions beyond a diameter, marking the closure of the near wake. The results of Calvert (1972) for a solid sphere at $R e=3.8 \times 10^{3}$ are also shown in Fig. 13 for comparison. It appears that in spite of ventilation, the structure of the wake is not strongly altered at subcritical $R e$. The differences with the results of Calvert are due to increased $R e$ of present results.

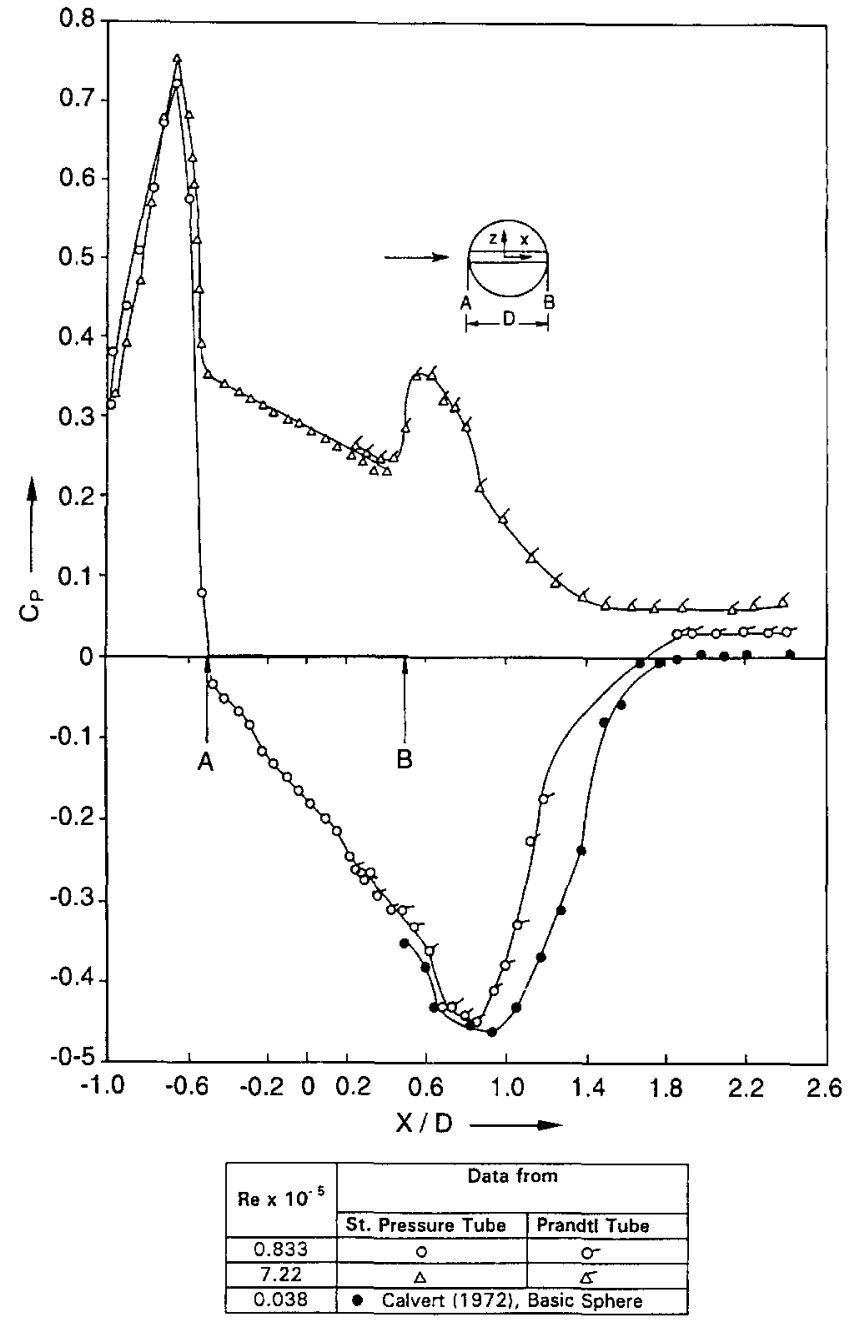

Fig. 13. Variation of static pressure along the centreline of the vented sphere

At supercritical $R e$, acceleration through the duct is reduced as a result of increased base pressure or reduced pressure differential. In contrast to that at lower $R e$, the base flow undergoes a rapid diffusion upon exit from the base, apparently due to the induced velocity of the secondary vortex ring (Fig. 5) and the static pressure reaches a maximum of $C p \cong 0.35$ at about $0.1 D$ from the base which is higher than the base pressure. The occurrence of positive pressures all over the flowfield is notable.

\section{7}

\section{Centreline velocity measurements}

Figure 14 shows the variation of velocity along the centreline of the vented sphere measured using a Prandtl tube at typical velocities in subcritical and supercritical range of $R e$. At subcritical $R e$, the vent flow is brought to rest within the dead water region. The vent mass is then deflected asymmetrically upstream of this stagnation point. Thus, even though all the vent mass was introduced at the centre of the sphere, very little or no mass flow emerges from the centre of the near wake. The vent mass travels upstream along with the reverse flow of the near wake and emerges along the separated shear layer. The phenomena observed is similar to that reported by Wong 


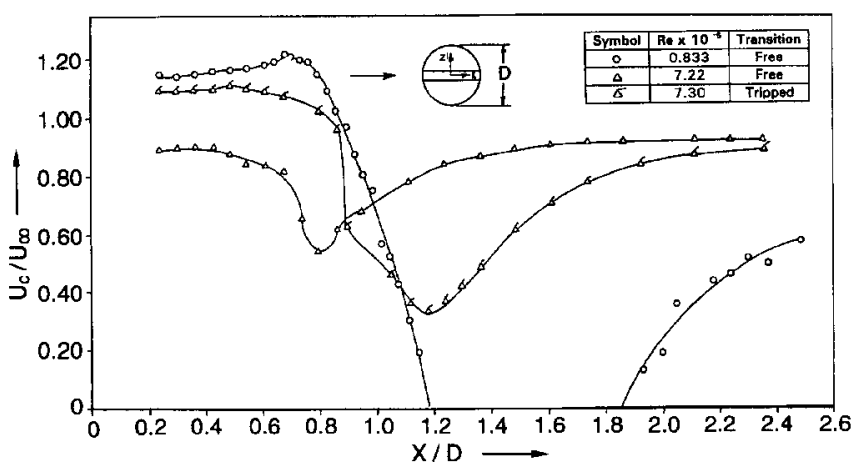

Fig. 14. Variation of velocity along the centreline of the vented sphere

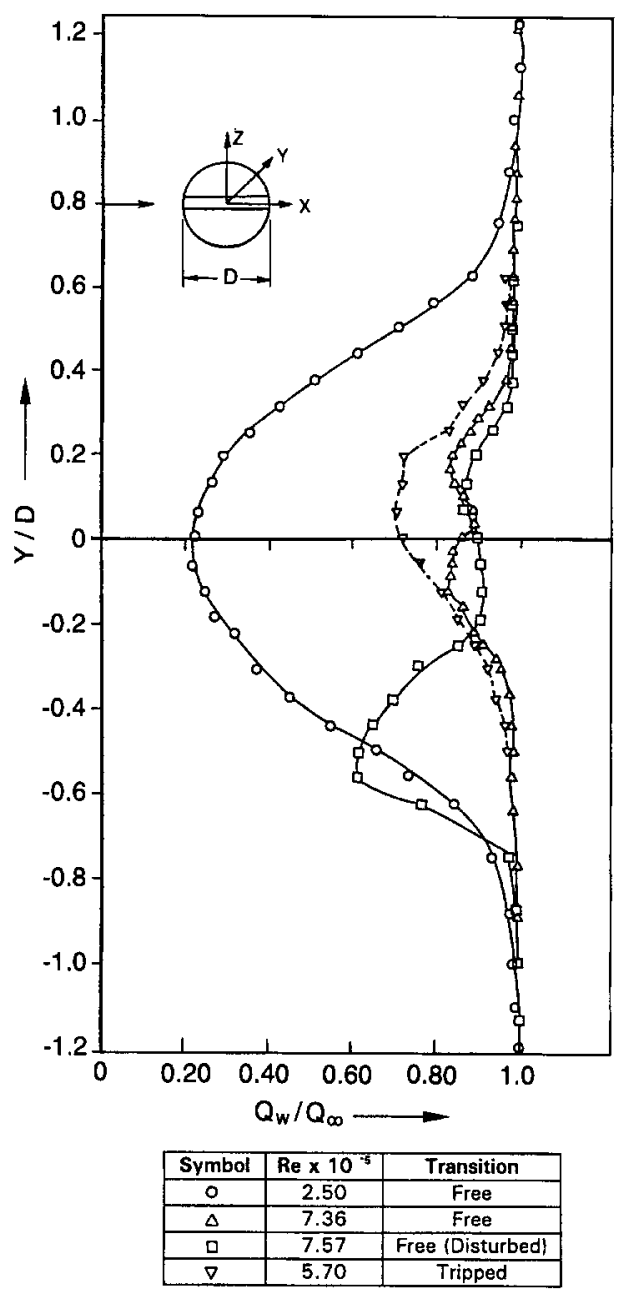

Fig. 15. Wake profile measured at $(x / D)=2.5,(z / D)=0.0$ behind the base of the vented sphere

(1985) for vented cylinder base flows. Kim and Durbin (1988) have identified two dominant modes of unsteadiness in the wake of the basic sphere in the range of $\operatorname{Re} 500$ to 60,000 . These are associated with the small scale instability of the separating shear layer (Kelvin-Helmholtz instability) and the large scale instability of the wake (vortex shedding) similar to that behind a cylinder as identified by Péterka and Richardson (1969).
Response of the internal jet seems to be governed by the large scale instability of the wake. The velocity measurements shows two stagnation points which correspond to the stagnation of the vent flow and the closure of the near wake. Reverse flow exists in the region within these two locations, as shown in Fig. 5.

At supercritical $R e$, positive velocity is observed all along the centreline, indicating that the vent flow has broken through the near wake. Diffusion of the vent flow caused by the secondary vortex ring (Fig. 5) reduces the centreline velocity to $0.55 U_{\infty}$ at about $0.3 D$ behind the base as compared to $0.9 U_{\infty}$ at the exit.

\section{8}

\section{Measurements in the wake of the vented sphere}

Figure 15 shows wake profiles at typical velocities in subcritical and supercritical range of $R e$. The effect of a $1.2 \mathrm{~mm}$ diameter trip wire at $70^{\circ}$ and the effect of a roughness element are also shown. At subcritical $R e$, the wake and the momentum defect are large despite ventilation since the vent flow is unable to split the wake. The width of the wake is unchanged since the separation of flow is unaltered. At supercritical $R e$, the profile clearly shows the effect of mass addition around the centre. Momentum losses are concentrated around an annular ring about the centreline, which also indicates that the wake has been symmetrically split by the vent flow. The resulting stream surface at the near wake aerodynamically streamlines the base of the sphere. Presence of a roughness element is shown to destroy the vortex structure, as discussed in 3.4 above resulting in a flow structure similar to that of the basic sphere as depicted in Fig. 5. Consequently, the wake is rendered asymmetric. The tripping wire completely destroys the vortex structure, advances turbulent separation and the drag reduction vanishes. The profile shows the wake of the tripped vented sphere to be much wider than that of the untripped vented sphere.

\section{4}

\section{Conclusions}

At supercritical $R e$ in an incompressible flow, the pressure drag of a sphere can be nearly nullified by ventilation. This is achieved by increased base pressures in the separated flow region close to the base. The vent flow at high $R e$ breaks through the near wake and brings about symmetry in the flow. When the internal shear is increased by tripping the internal duct, the external flow is accelerated to velocities beyond that due to potential flow around a solid sphere. The observations can be explained by a flow model in which the near wake is streamlined by a pair of counterrotating vortex rings trapped around a region of potential flow. A roughness element can be made to partially destroy the vortex system at the base and result in a steady asymmetric wake. A $1.2 \mathrm{~mm}$ diameter wire placed at $70^{\circ}$ completely destroys the vortex rings by overtripping the boundary layer. Simultaneously, separation of the boundary layer is advanced, base pressure recovery reduced and pressure drag increased.

At subcritical $R e$, ventilation marginally increases the pressures all over the flowfield. Since the large pressure differential between windward and leeward sides is hardly altered, there is no significant drag reduction. The internal flow accelerates to velocities close to the free stream value and then 
stagnates in the near wake. The main features of the basic sphere are unaltered in spite of ventilation. The upstream effects of ventilation are higher at subcritical $R e$ than at supercritical $R e$.

\section{References}

Achenbach E (1972) Experiments on the flow past spheres at high Reynolds numbers. J Fluid Mech 54: 565-575

Achenbach E (1974) The effects of surface roughness and tunnel blockage on the flow past spheres. J Fluid Mech 65: 113-125

Bearman PW (1967) The effect of base bleed on the flow behind a two-dimensional model with a blunt trailing edge. The Aeronautical Quarterly 18: 207-224

Calvert JR (1972) Some experiments on the flow past a sphere. Aero J Roy Aero Soc 76: 248-250

Dallmann U; Schewe G (1987) On topological changes of separating flow structures at transition Reynolds numbers. AIAA-87-1266

Fage A (1936) Experiments on a sphere at critical Reynolds numbers ARC R \& M No: 1766

Kim HJ; Durbin PA (1988) Observations of the frequencies in a sphere wake and of drag increase by acoustic excitation. Physics of Fluids 31: $3260-3265$

Maxworthy T (1969) Experiments on the flow around a sphere at high Reynolds numbers. J Appl Mech Trans ASME E36: 598-607

Meier GEA; Suryanarayana GK; Pauer H (1990) Widerstandsverminderung durch Ventilation. DGLR Bericht 90-06, 311-315

Meier GEA; Suryanarayana GK; Pauer H (1991) Bluff-body drag reduction by passive ventilation. First European Conference on Fluid Mechanics EUROMECH, Cambridge, UK, 16-20 Sept.

Peterka JA; Richardson PD (1969) Effects of sound on separated flows. J Fluid Mech 37: 265-287
Raithby GD; Eckert ERG (1968) The effect of support position and turbulence intensity on the flow near the surface of a sphere. Wärme und Stoffübertrag 1 2: 87--94

Roshko A (1954) On the drag and shedding frequency of two-dimensional bluff bodies. NACA TN 3169

Suryanarayana GK; Pauer H; Meier GEA (1992) Passive control of the wake of a sphere by ventilation. Proc. IUTAM Conference on Bluff-Body Wakes, Dynamics and Instabilities. Springer-Verlag 91-94

Suryanarayana GK; Pauer H; Meier GEA (1993) Bluff-body drag reduction by passive ventilation. Exp Fluids 16: 73-81

Taneda S (1978) Visual observations of the flow past a sphere at Reynolds numbers between $10^{4}$ and $10^{6}$. J Fluid Mech 85: 187-192

Wong HY (1985) Wake stabilisation by the action of base bleed. J Fluids Eng Trans ASME 107: 378-384

Wood CJ (1964) The effect of base bleed on a periodic wake. J Roy Aero Soc 68: 477-482

Wood CJ (1967) Visualisation of an incompressible wake with base bleed. J Fluid Mech 29: 259-272

Zhdanov VL; Eckelmann H (1990) The effects of jet bleed on base pressure distribution, shedding frequency and mean velocity profiles in the wake behind a two-dimensional model. Max-Planck-Institute for Fluid Research Rep. no. 9, Göttingen, Germany

Zhdanov VL; Eckelmann H (1991) An experimental investigation of the wake turbulent structure behind a two-dimensional blunt model. Max-Planck-Institute for Fluid Research Rep. no. 9, Göttingen, Germany

Zhdanov VL; Eckelmann H; Stasicki B (1992) Base bleed efficiency criterion. Proc IUTAM Conference on Bluff-Body Wakes, Dynamics and Instabilities. 221-225. Springer-Verlag 